Praneetha Thulasi, BA

Clare L. Fraser, MD

Valérie Biousse, MD

David W. Wright, MD

Nancy J. Newman, MD

Beau B. Bruce, MD, MS

Correspondence to

Dr. Bruce:

bbbruce@emory.edu

\title{
Nonmydriatic ocular fundus photography among headache patients in an emergency department
}

\section{$[\square]$}

\section{ABSTRACT}

Objectives: Determine the frequency of and the predictive factors for abnormal ocular fundus findings among emergency department (ED) headache patients.

Methods: Cross-sectional study of prospectively enrolled adult patients presenting to our ED with a chief complaint of headache. Ocular fundus photographs were obtained using a nonmydriatic fundus camera that does not require pupillary dilation. Demographic and neuroimaging information was collected. Photographs were reviewed independently by 2 neuroophthalmologists for findings relevant to acute care. The results were analyzed using univariate statistics and logistic regression modeling.

Results: We included 497 patients (median age: 40 years, 73\% women), among whom 42 (8.5\%, 95\% confidence interval: 6\%-11\%) had ocular fundus abnormalities. Of these 42 patients, 12 had disc edema, 9 had optic nerve pallor, 6 had grade III/IV hypertensive retinopathy, and 15 had isolated retinal hemorrhages. Body mass index $\geq 35 \mathrm{~kg} / \mathrm{m}^{2}$ (odds ratio [OR]: $2.3, p=0.02$ ), younger age (OR: 0.7 per 10-year increase, $p=0.02$ ), and higher mean arterial blood pressure (OR: 1.3 per 10-mm Hg increase, $p=0.003$ ) were predictive of abnormal retinal photography. Patients with an abnormal fundus had a higher percentage of hospital admission ( $21 \%$ vs $10 \%, p=0.04)$. Among the 34 patients with abnormal ocular fundi who had brain imaging, 14 (41\%) had normal imaging.

Conclusions: Ocular fundus abnormalities were found in $8.5 \%$ of patients with headache presenting to our ED. Predictors of abnormal funduscopic findings included higher body mass index, younger age, and higher blood pressure. Our study confirms the importance of funduscopic examination in patients with headache, particularly in the ED, and reaffirms the utility of nonmydriatic fundus photography in this setting. Neurology ${ }^{\circledR}$ 2013;80:432-437

\section{GLOSSARY}

BMI = body mass index; ED = emergency department; EMR = electronic medical record; FOTO-ED = Fundus photography vs Ophthalmoscopy Trial Outcomes in the Emergency Department; HIPAA = Health Insurance Portability and Accountability Act; IIH = idiopathic intracranial hypertension; IQR = interquartile range; $\mathbf{O R}=$ odds ratio.

In the emergency department (ED), headache is the fourth most common chief complaint among all patients and the single most common neurologic chief complaint. ${ }^{1}$ More than 3 million ED visits for headache occurred in the United States during 2008, ${ }^{2}$ and it is estimated that 3\% to 5\% of the general population has visited an ED for headache. ${ }^{3}$ The vast majority of these headaches are primary, requiring only symptomatic relief., ${ }^{2,5}$ However, approximately $2 \%$ of all patients with headache and more than $18 \%$ of patients with sudden, severe headaches have a serious underlying cause. ${ }^{4,6-9}$ The frequency of secondary headaches also increases with age, occurring in almost $15 \%$ of headache sufferers older than 75 years. ${ }^{4}$

The ED physician has the difficult task of discriminating between nonemergent headaches and those requiring acute care. Examination of the ocular fundus is one aspect of the physical examination that helps screen for potential "red flags," such as papilledema, that suggest a more ominous cause of headache. ${ }^{10,11}$ Unfortunately, funduscopy, performed infrequently in acute care settings such as the ED, is also not accurately interpreted by nonophthalmology personnel. ${ }^{10}$ Our goal was to

From the Departments of Ophthalmology (P.T., C.L.F., V.B., N.J.N., B.B.B.), Emergency Medicine (D.W.W.), Neurology (V.B., N.J.N., B.B.B.), and Neurological Surgery (N.J.N.), Emory University School of Medicine; and Rollins School of Public Health and Laney Graduate School (B.B.B.), Emory University, Atlanta, GA.

Go to Neurology.org for full disclosures. Funding information and disclosures deemed relevant by the authors, if any, are provided at the end of the article. 
examine the subset of patients from the Fundus photography vs Ophthalmoscopy Trial Outcomes in the Emergency Department (FOTOED) study ${ }^{10,11}$ who presented with headaches, in order to characterize the abnormal ocular fundus findings seen and to determine the factors predictive of abnormal ocular fundus findings among headache patients presenting to the ED.

METHODS Standard protocol approvals, registrations, and patient consents. The study was approved by our Institutional Review Board. Patients were screened under a partial Health Insurance Portability and Accountability Act (HIPAA) waiver. Informed consent was obtained from all enrolled study participants using a modified written consent procedure.

Study conduct. We conducted the FOTO-ED study between April 2009 and August 2011 (clinicaltrials.gov: NCT00873613). The study was conducted in 2 phases. The first phase evaluated the routine clinical use of direct ophthalmoscopy by ED physicians, ${ }^{10,11}$ whereas the second phase evaluated the routine use of nonmydriatic ocular fundus photography as interpreted by the $\mathrm{ED}$ physicians. In both phases, all patients had nonmydriatic ocular fundus photographs obtained. Between the 2 active FOTO-ED enrollment phases, additional patients meeting all relevant study criteria were included (e.g., patients enrolled during the lead-in period of the second phase).

The inclusion criteria for the FOTO-ED study were adult patients presenting to the $\mathrm{ED}$ with a chief complaint of one or more of the following: headaches, focal neurologic deficits, diastolic blood pressure $\geq 120 \mathrm{~mm} \mathrm{Hg}$, and acute visual changes. The chief complaints were elicited from the patients in the usual fashion (without systematic screening for headache) and entered into the electronic medical record (EMR) by the triage staff. Patients were excluded if they were unable to sit up, refused to participate, had altered mental status or were otherwise unable to consent, or were too ill to participate in the study. Nurse practitioners were available to photograph patients from $7 \mathrm{AM}$ to 10 PM daily during the first phase and a medical student was similarly available from 8 AM to 6 PM on weekdays during the second phase. Patients who presented to the ED outside of these hours could be included if they were still present in the ED during these hours. Additionally, several randomly chosen night and weekend shifts were included during the second phase. The present analysis describes a cross-sectional study of the subset of patients presenting with a chief complaint of headache. Patients were identified as potentially eligible for enrollment based on the symptoms entered into the EMR at triage. During the first phase, this required active surveillance of the triage log by a study team member, but after the first phase, patients were automatically flagged for consideration for fundus photography by the EMR when their chief complaint was entered. Active surveillance of the ED's census by study staff identified potentially eligible patients who did not trigger the automated process. Additionally, the log of patients who triggered screening was reviewed for patients who were inadvertently not included.

Patient demographics, including age, gender, race, presenting vital signs, height, and weight, were prospectively collected in the ED. Documentation of direct ophthalmoscopy by emergency physicians, medical history, medication use, imaging, consultations, and other interventions were obtained from chart review by 2 members of the study team masked to fundus photography results. Imaging interpretations were based on final radiology reports.

Photographs of the posterior pole of the ocular fundus (optic disc, macula, and major retinal vessels) were obtained from both eyes of enrolled patients at presentation by trained nurse practitioners or a medical student using a commercially available, Food and Drug Administration-approved, nonmydriatic ocular fundus camera (Kowa nonmyd- $\alpha-D$ 5-megapixel camera; Kowa Optimed, Inc., Torrance, CA) according to previously described methods. ${ }^{10}$

The images were automatically electronically transferred to a HIPAA-compliant database for review by 2 neuro-ophthalmologists who were masked to all patient data, including chief complaint. These reviewers rated the images for the presence or absence of relevant ocular fundus abnormalities using a standardized case report form. In any case in which there remained diagnostic uncertainty, a third neuroophthalmologist made the determination of whether an abnormality was present or absent, masked to patient data but aware of the finding disagreed on by the other 2 reviewers. If there still remained uncertainty, an in-office examination was arranged.

The primary outcome measure was the detection of ocular funduscopic findings relevant to acute patient care in the ED (i.e., findings that should or could change the patient's acute evaluation, treatment, and disposition). Relevant ocular fundus abnormalities were defined a priori as optic disc edema, optic disc pallor, retinal vascular occlusions, intraocular hemorrhages, and grade III/IV hypertensive retinopathy. Four patients $(<1 \%)$ had photographs that were ungradable; these patients were included in the analysis as having no relevant findings. Detailed information about quality and interobserver variability in the FOTO-ED study was reported previously. ${ }^{12}$

Data analysis. Statistical analysis was performed using the R:A language and environment for statistical computing ( $R$ Foundation for Statistical Computing, http://www.R-project.org). Medians and interquartile ranges (IQRs) were reported for continuous data and percentages were reported for categorical data. Medians were compared using the Mann-Whitney $U$ test and proportions were compared using $\chi^{2}$ or Fisher exact tests, as appropriate. Two-tailed $p$ values $<0.05$ were considered statistically significant.

Binary logistic regression was performed, with the presence of relevant abnormalities by fundus photography as the outcome and with age, sex, race, body mass index (BMI), and mean arterial blood pressure (calculated according to the method of Razminia et al. ${ }^{13}$ ) as multiple predictors of interest. Model selection was performed using the method of Kleinbaum and Klein. ${ }^{14}$ Multinomial logistic regression was performed, with the outcome being the fundus diagnosis (disc edema; grade III/IV hypertensive retinopathy or intraocular hemorrhages; optic disc pallor; or normal) with the same predictors identified from the final binary logistic model (age, BMI $\geq 35 \mathrm{~kg} / \mathrm{m}^{2}$, and mean arterial pressure).

RESULTS Of 820 patients enrolled, 497 patients $(61 \%)$ had a chief complaint of headache. The median age was 40 years (IQR: $32-55$ years). Three hundred sixty-three (73\%) were women, and $265(53 \%)$ were black. During the second phase (when exclusions were actively recorded), 212 headache patients ( $43 \%$ of 497\%) were enrolled (206 identified via the automated system, 6 via active surveillance by study staff) from among 227 eligible (11 refused, 3 eligible missed by study staff, and 1 unavailable at a diagnostic test).

Ocular fundus abnormalities were found on photographs of 42 of the 497 headache patients $(8.5 \%$; 95\% confidence interval: $6 \%-11 \%): 15$ of $42(36 \%)$ isolated retinal hemorrhages; 12 of 42 (29\%) disc edema, of which 11 were bilateral (figure); 9 of 42 (21\%) optic nerve pallor; and 6 of 42 (14\%) grade III/IV hypertensive retinopathy. Thirty of 400 patients with an isolated 
Figure

Nonmydriatic fundus photographs of emergency department patients

complaining of headache

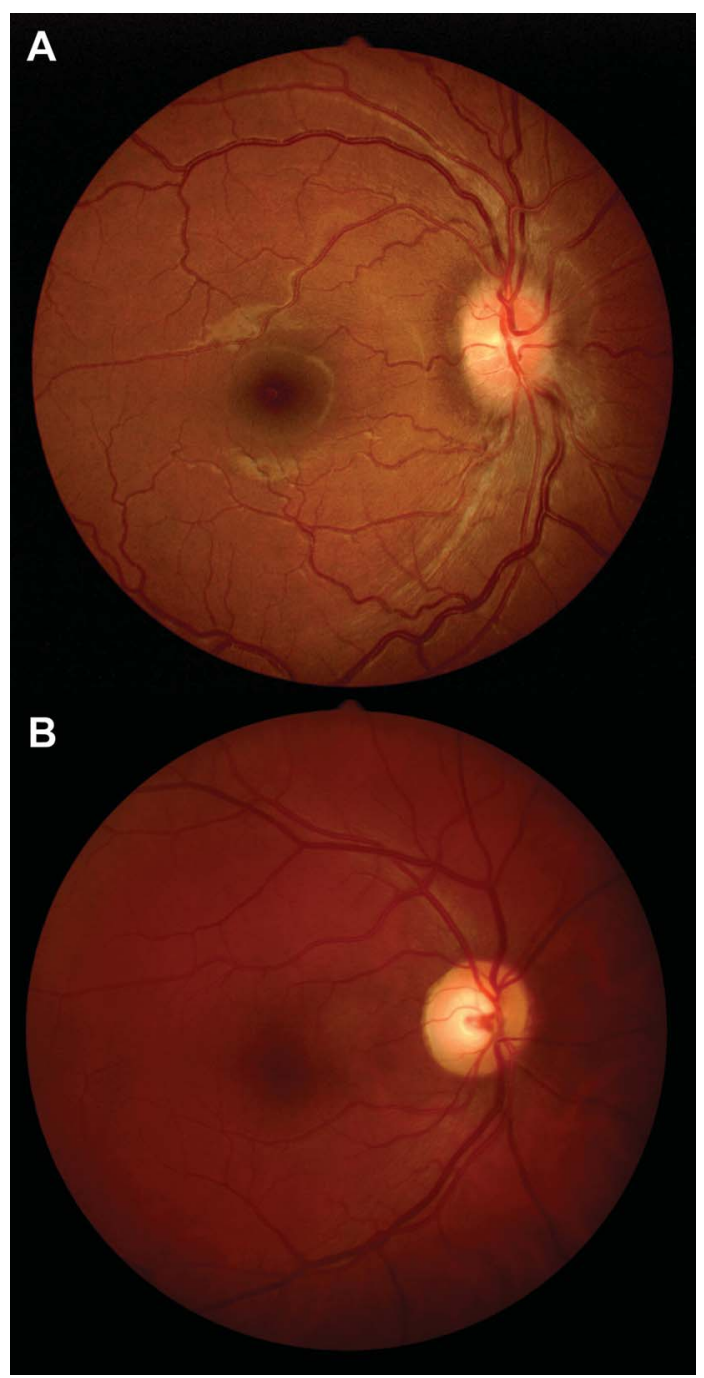

(A) Optic disc edema. (B) Normal ocular fundus photograph after a recent neurosurgical shunting procedure, reported by the emergency department physician as being helpfu in the evaluation of the case because it was normal.

chief complaint of headache had fundus abnormalities, and 12 among the 97 remaining patients who had headache plus one or more additional chief complaints had abnormal findings ( $8 \%$ vs $12 \%, p=0.17$ ).

Patients with abnormal fundus photographs were younger (median age: 37 vs 42 years, $p=0.05$ ) and had a higher BMI (32 vs $27 \mathrm{~kg} / \mathrm{m}^{2} ; p=0.04$ ) than patients with normal ocular fundi (table 1). There were also trends toward more frequent black race $(67 \%$ vs $52 \%, p=0.07)$ and a higher mean arterial pressure (108 vs $103 \mathrm{~mm} \mathrm{Hg}, p=0.06$ ) among those with an abnormal ocular fundus.

Patients with an abnormal fundus were more likely to have brain imaging obtained $(81 \%$ vs $65 \%, p=0.04$ ), with $41 \%$ of these imaging studies interpreted as normal. Patients with an abnormal fundus were also more likely to have ophthalmology, neurology, or other consultations requested $55 \%$ vs $32 \%, p=0.003)$ and to be admitted to the hospital ( $21 \%$ vs $10 \%, p=0.04$; table 1$)$.

Twenty-eight of the 228 headache patients enrolled in the first phase (12\%) had direct ophthalmoscopy performed by ED physicians. Four of these 28 patients had relevant abnormalities ( 2 optic disc edema, 2 isolated retinal hemorrhages), none of which were detected by the ED physician examination. One hundred forty-six of the 206 headache patients enrolled in the second phase (71\%) had fundus photographs reviewed by the ED physicians. Eight of the 19 relevant findings (42\%) that occurred during this phase among headache patients were identified by the ED physicians who reviewed 17 of these 19 patients' photographs: 3 with optic disc edema, 2 with optic disc pallor, 2 with isolated retinal hemorrhages, and 1 with grade III/IV hypertensive retinopathy. The remaining unidentified relevant findings were 5 optic disc pallor, 1 optic disc edema, 2 isolated retinal hemorrhages, and 1 grade III/IV hypertensive retinopathy.

Logistic regression showed that higher BMI, younger age, and higher mean arterial pressure were independent predictors for abnormal ocular fundus findings among patients with headache (table 2). Neither race nor sex were significant predictors of abnormal funduscopic findings, nor were they confounders of the relationship between the other predictors and the outcome. There was no evidence of multiplicative interaction with respect to the $\log$ OR. Multinomial logistic regression found that younger age (OR 3.2 per 10-year age decrease, $p=0.002)$ and $\mathrm{BMI} \geq 35 \mathrm{~kg} / \mathrm{m}^{2}$ (OR 1.3 , $p=0.045)$ predicted the presence of disc edema; higher mean arterial pressure (OR 1.5 per $10-\mathrm{mm} \mathrm{Hg}$ increase, $p=0.0002)$ predicted the presence of intraocular hemorrhage or grade III/IV hypertensive retinopathy; and BMI $\geq 35 \mathrm{~kg} / \mathrm{m}^{2}$ (OR 1.5, $\left.p=0.03\right)$ predicted the presence of optic disc pallor.

DISCUSSION Our study found that $8.5 \%$ of patients presenting to the ED with a chief complaint of headache had relevant ocular fundus abnormalities detected by nonmydriatic ocular fundus photography. This suggests that diagnostically relevant ocular fundus findings may be present in more than 250,000 of the over 3 million patients who visit an ED for headache each year in the United States. ${ }^{2}$ The relative high frequency of these ocular findings, often associated with otherwise unremarkable physical examinations, and in many cases normal neuroimaging studies ( $41 \%$ of the patients with abnormal fundi in our study), emphasizes the important role of ophthalmoscopic examination in the evaluation of patients with headache.

Although there is a substantial body of literature on the retinal microvascular changes in migraineurs, there 
Table 1 Univariate analysis of the characteristics of patients with headache by status of ocular fundus

\begin{tabular}{|c|c|c|c|c|c|}
\hline & \multicolumn{4}{|l|}{ Ocular fundus } & \multirow[b]{3}{*}{ p Value } \\
\hline & \multicolumn{2}{|l|}{ Abnormal $(n=42)$} & \multicolumn{2}{|l|}{ Normal $(n=455)$} & \\
\hline & Count or median & $\%$ or IQR & Count or median & $\%$ or IQR & \\
\hline \multicolumn{6}{|l|}{ Demographics } \\
\hline Women & 29 & $69 \%$ & 334 & $73 \%$ & 0.54 \\
\hline Age, y & 37 & $27-49$ & 42 & $31-54$ & 0.05 \\
\hline Black race & 28 & $67 \%$ & 237 & $52 \%$ & 0.07 \\
\hline Body mass index, $\mathrm{kg} / \mathrm{m}^{2}$ & 32 & $24-40$ & 27 & $24-33$ & 0.04 \\
\hline Mean arterial pressure, $\mathrm{mm} \mathrm{Hg}$ & 108 & $99-125$ & 103 & $94-114$ & 0.06 \\
\hline \multicolumn{6}{|l|}{ ED stay characteristics } \\
\hline ED length of stay, $d$ & 0.42 & $0.2-0.5$ & 0.39 & $0.2-0.4$ & 0.58 \\
\hline Neuroimaging obtained & 34 & $81 \%$ & 296 & $65 \%$ & 0.04 \\
\hline Normal neuroimaging ${ }^{a}$ & $14 / 34^{b}$ & $41 \%$ & 237/296 & $80 \%$ & $<0.001$ \\
\hline Consultation obtained & 23 & $55 \%$ & 147 & $32 \%$ & 0.003 \\
\hline Hospital admission & 9 & $21 \%$ & 46 & $10 \%$ & 0.04 \\
\hline
\end{tabular}

Abbreviations: ED = emergency department; IQR = interquartile range.

${ }^{a}$ Among patients in whom neuroimaging was obtained.

${ }^{\mathrm{b}}$ Twenty-six patients had CT only, 2 patients had MRI only, and 6 had both CT and MRI.

have been no prior studies, to our knowledge, systematically evaluating headache patients for the prevalence of abnormal ocular fundus findings. ${ }^{15-19}$ One prior study reported that an "abnormal cranial nerve examination" significantly increased the odds of secondary headache, but it is unknown whether direct observation of the optic nerve by funduscopic examination was included, and how many of the reported "cranial nerve abnormalities" involved the optic nerve.?

Despite the known potential for abnormal ocular findings in patients with visual and neurologic complaints or elevated blood pressure, ED physicians do not systematically perform direct ophthalmoscopy. ${ }^{11}$ Indeed, during the first phase of the FOTO-ED study, ED physicians examined the ocular fundi by direct ophthalmoscopy in only $12 \%$ of headache patients. Although direct ophthalmoscopy is a difficult skill to perform and master, ${ }^{20,21}$ the high potential for devastating patient outcomes and serious medicolegal consequences suggest that the status quo is not acceptable. Alternative techniques for examining the ocular fundus

\begin{tabular}{|lccc|}
\hline Table 2 & \multicolumn{2}{l}{$\begin{array}{l}\text { Final multivariate logistic regression model with abnormal fundus } \\
\text { finding as outcome }\end{array}$} \\
& OR & $95 \% \mathrm{Cl}$ & $\mathrm{p}$ Value \\
Age, per $10-\mathrm{y}$ increase & 0.7 & $0.57-0.95$ & 0.02 \\
Body mass index, $\geq 35 \mathrm{~kg} / \mathrm{m}^{2}$ & 2.3 & $1.16-4.50$ & 0.02 \\
Mean arterial pressure, per $10-\mathrm{mm} \mathrm{Hg}$ increase & 1.3 & $1.09-1.55$ & 0.003 \\
\end{tabular}

Abbreviations: $\mathrm{Cl}=$ confidence interval; $\mathrm{OR}=$ odds ratio. may be needed, and we have shown that nonmydriatic fundus photography is a feasible method of ocular fundus examination in the busy ED setting. ${ }^{10,11}$

Optic disc pallor represented approximately $20 \%$ of the ocular fundus abnormalities observed in this study. Optic disc pallor can result from any process damaging the anterior visual pathways, but only becomes visible 4 to 6 weeks after injury. Although optic atrophy might not be considered an important finding acutely in the $\mathrm{ED}$, its identification is very relevant to the management of headache patients because it might reflect chronically increased intracranial pressure with end-stage papilledema or chronic anterior visual pathway compression from an intracranial mass lesion. Either mechanism may explain the increased frequency of optic nerve pallor we found among patients with a high BMI because obesity is associated with idiopathic intracranial hypertension (IIH) and with an increased risk of CNS tumors, particularly meningiomas that frequently affect the anterior visual pathways. ${ }^{22,23}$

Optic disc edema, representing nearly a third of the abnormalities found, was observed more frequently than optic disc pallor. Among patients who present to the ED with headache, this is particularly relevant because optic disc edema frequently indicates increased intracranial pressure, especially when bilateral, as was seen in more than $90 \%$ of the cases. We found that younger age and obesity were independently predictive of optic disc edema, which may reflect patients with papilledema from underlying $\mathrm{IIH}$, typically a disorder of young, obese individuals. Because patients with IIH often present with isolated headache, and by definition have 
normal neuroimaging, examination of the ocular fundus is the primary way of identifying this subgroup of pathologic headaches. An increased number of abnormal fundus findings among younger headache patients also stresses the independent relevance of the ocular fundus examination in the evaluation of headache patients, because it runs counter to the overall trend of secondary headache occurring more frequently in older patients. ${ }^{4,24}$

The largest group of optic nerve abnormalities detected $(36 \%)$ constituted isolated retinal hemorrhages. If we presume that the majority of these were secondary to hypertension, ${ }^{25,26}$ and combine them with the $14 \%$ of the abnormal fundi with grade III/IV hypertensive retinopathy, then approximately half of the headache patients with abnormal ocular fundi had findings attributable to hypertension. We found that higher mean arterial pressure was predictive of abnormal funduscopic findings, particularly the presence of retinal hemorrhages and grade III/IV hypertensive retinopathy, among headache patients. Our findings might seem surprising because large and well-conducted, population-based studies and the consensus of the International Headache Society suggest no association between mild to moderate hypertension and headache, despite a popular perception to the contrary. ${ }^{5,27}$ Indeed, one study found that a high pulse pressure was protective against headache. ${ }^{28}$ However, headache is one of the most common presenting symptoms of hypertensive urgencies, and other evidence has associated more severe degrees of hypertension with headache. ${ }^{29,30}$ Thus, the high frequency of hypertensive-related changes in our study is unlikely to be coincidental. In addition, we previously found that malignant hypertensive retinopathy occurred more frequently in younger patients (irrespective of the presence of headache) and that the current blood pressure screening threshold for end-organ damage among patients with severely increased blood pressure (i.e., exceeding 180/120 mm Hg, as defined by the Seventh Report of the Joint National Committee [JNC7] on Prevention, Detection, Evaluation, and Treatment of High Blood Pressure) is not low enough to capture all the important ocular findings likely related to hypertensive damage. ${ }^{26}$ Therefore, the presence of headache may be helpful in determining which hypertensive patients to screen for ophthalmic end-organ damage, an important, but not infrequently isolated, sign of malignant hypertension. ${ }^{31}$

An abnormal ocular fundus examination also had implications for the disposition of patients in the ED. Specifically, those patients with fundus abnormalities received consultation and hospital admission more often, likely because they had more severe underlying disorders causing their headache. In addition, some patients needed to be called back to the ED after discharge because relevant findings (e.g., papilledema and malignant hypertension) were identified only by neuroophthalmologist review of fundus photographs. Clearly, more consistent and accurate ocular fundus examinations will directly alter the management of some headache patients' ED care.

One limitation of our study was its performance in a tertiary ED that routinely evaluates patients with more severe pathology, and thus the prevalence of abnormal funduscopic findings may be higher than would be expected in a community setting. Furthermore, the design of the second phase of the FOTO-ED study provided the ED physicians access to fundus photographs, perhaps resulting in a higher rate of consultations and hospital admissions than in the first phase. However, because the photographs were reviewed promptly in both phases, with physicians notified of missed urgent findings, it is unlikely that ED-physician interpretation of the images affected the admission rate. Although our sample had convenience aspects because of the limited availability of study staff and because screen failures were only documented during the second phase, the similar rate of relevant abnormalities in the second phase (9.4\%) compared with the overall study (8.5\%), and the inclusion of $93 \%$ of the eligible population during the second phase, would suggest that our sample is representative of patients presenting with headache to our institution's ED. Even if we assume that patients presenting during the nights and weekends were substantially different than patients presenting during weekdays (an assumption not supported by our nights and weekend data), our findings would still have important implications for ED patient care.

Ocular funduscopic abnormalities occurred in 8.5\% of patients presenting with headaches to the ED. Younger age, higher BMI, and higher blood pressure were predictive of the presence of ocular fundus abnormalities. Funduscopic examination provides key information during the evaluation of headache patients beyond what is provided by the remainder of the physical examination and neuroimaging. The examination of the ocular fundus seems to be particularly relevant for younger patients, who are often presumed to have primary headaches. The recent availability of nonmydriatic digital cameras, which allow easy and reliable ocular fundus imaging in the ED and potential telemedical applications, facilitates the examination of the ocular fundus in patients with headache.

\section{AUTHOR CONTRIBUTIONS}

Praneetha Thulasi: study design; analysis/interpretation of data; drafting/ revising manuscript for content. Clare L. Fraser: analysis of data; revising manuscript for content. Valérie Biousse: study design; interpretation of data; revising manuscript for content. David W. Wright: study design; revising manuscript for content. Nancy J. Newman: study design; interpretation of data; revising manuscript for content. Beau B. Bruce: study design; analysis/ interpretation of data; drafting/revising manuscript for content.

\section{STUDY FUNDING}

This study was supported in part by an unrestricted departmental grant (Department of Ophthalmology) from Research to Prevent Blindness, Inc., 
New York, the NIH/NEI core grant P30-EY06360 (Department of Ophthalmology), and NIH grants K23-EY019341, KL2-RR025009, and UL1RR025008.

\section{DISCLOSURE}

P. Thulasi reports no disclosures. C. Fraser received the RANZCO Eye Foundation Scholarship and the Sydney Eye Alumni Travelling Fellowship Grant. V. Biousse received research support from the NIH/PHS (UL1RR025008). D. Wright received research support from NIH/PHS (KL2RR025009). N. Newman received the Research to Prevent Blindness and a Lew R. Wasserman Merit Award; and has provided expert testimony on the topic of papilledema. B. Bruce receives research support from the NIH/NEI (K23-EY019341); received research support from NIH/PHS (KL2-RR025009, UL1-RR025008) and the Knights Templar Eye Foundation. Go to Neurology.org for full disclosures.

Received May 8, 2012. Accepted in final form September 26, 2012.

\section{REFERENCES}

1. Pitts SR, Niska RW, Xu J, Burt CW. National Hospital Ambulatory Medical Care Survey: 2006 emergency department summary. Natl Health Stat Report 2008;(7):1-38.

2. Lucado J, Paez K, Elixhauser A. Headaches in U.S. Hospitals and Emergency Departments, 2008: HCUP Statistical Brief \#111. Rockville: Agency for Healthcare Research and Quality; 2011.

3. Linet MS, Stewart WF, Celentano DD, Ziegler D, Sprecher M. An epidemiologic study of headache among adolescents and young adults. JAMA 1989;261:2211-2216.

4. Goldstein JN, Camargo CA Jr, Pelletier AJ, Edlow JA. Headache in United States emergency departments: demographics, work-up and frequency of pathological diagnoses. Cephalalgia 2006;26:684-690.

5. Olesen J. The international classification of headache disorders. 2nd edition (ICHD-II). Rev Neurol (Paris) 2005;161:689-691.

6. Fodden DI, Peatfield RC, Milsom PL. Beware the patient with a headache in the accident and emergency department. Arch Emerg Med 1989;6:7-12.

7. Landtblom AM, Fridriksson S, Boivie J, Hillman J, Johansson G, Johansson I. Sudden onset headache: a prospective study of features, incidence and causes. Cephalalgia 2002;22:354-360

8. Locker T, Mason S, Rigby A. Headache management-are we doing enough? An observational study of patients presenting with headache to the emergency department. Emerg Med J 2004;21:327-332.

9. Ramirez-Lassepas M, Espinosa CE, Cicero JJ, Johnston KL, Cipolle RJ, Barber DL. Predictors of intracranial pathologic findings in patients who seek emergency care because of headache. Arch Neurol 1997;54:1506-1509.

10. Bruce BB, Lamirel C, Biousse V, et al. Feasibility of nonmydriatic ocular fundus photography in the emergency department: phase I of the FOTO-ED study. Acad Emerg Med 2011;18:928-933.

11. Bruce BB, Lamirel C, Wright DW, et al. Nonmydriatic ocular fundus photography in the emergency department. N Engl J Med 2011;364:387-389.

12. Lamirel C, Bruce BB, Wright DW, Delaney KP, Newman NJ, Biousse V. Quality of nonmydriatic digital fundus photography obtained by nurse practitioners in the emergency department: the FOTO-ED study. Ophthalmology 2012;119:617-624.

13. Razminia M, Trivedi A, Molnar J, et al. Validation of a new formula for mean arterial pressure calculation: the new formula is superior to the standard formula. Catheter Cardiovasc Interv 2004;63:419-425.

14. Kleinbaum DG, Klein M. Logistic Regression: A Self-Learning Text, 2nd ed. New York: Springer-Verlag; 2002.

15. Gomi S, Gotoh F, Komatsumoto S, Ishikawa Y, Araki N, Hamada J. Sweating function and retinal vasomotor reactivity in migraine. Cephalalgia 1989;9:179-185.

16. Joffe SN. Retinal blood vessel diameter during migraine. S Afr Med J 1971;45:1215-1218.

17. Liew G, Campbell S, Klein R, et al. Ten-year longitudinal changes in retinal microvascular lesions: the atherosclerosis risk in communities study. Ophthalmology 2011;118:1612-1618.

18. Liew G, Mitchell P, Wong TY, Wang JJ. Retinal vascular caliber and migraine: the Blue Mountains Eye Study. Headache 2006;46:997-1004.

19. Rose KM, Wong TY, Carson AP, Couper DJ, Klein R, Sharrett AR. Migraine and retinal microvascular abnormalities: the Atherosclerosis Risk in Communities Study. Neurology 2007;68:1694-1700.

20. Benbassat J, Polak BC, Javitt JC. Objectives of teaching direct ophthalmoscopy to medical students. Acta Ophthalmol 2012;90:503-507.

21. Cordeiro MF, Jolly BC, Dacre JE. The effect of formal instruction in ophthalmoscopy on medical student performance. Med Teach 1993;15:321-325.

22. Benson VS, Pirie K, Green J, Casabonne D, Beral V. Lifestyle factors and primary glioma and meningioma tumours in the Million Women Study cohort. Br J Cancer 2008;99: 185-190.

23. Michaud DS, Bove G, Gallo V, et al. Anthropometric measures, physical activity, and risk of glioma and meningioma in a large prospective cohort study. Cancer Prev Res (Phila) 2011; 4:1385-1392.

24. Gilbert JW, Johnson KM, Larkin GL, Moore CL. Atraumatic headache in US emergency departments: recent trends in CT/MRI utilisation and factors associated with severe intracranial pathology. Emerg Med J 2012;29:576-581.

25. Henderson AD, Biousse V, Newman NJ, Lamirel C, Wright DW, Bruce BB. Grade III or IV hypertensive retinopathy in the setting of severely elevated blood pressure. West J Emerg Med Epub 2012 Feb 21.

26. Bruce BB, Lamirel C, Wright DW, Biousse V, Newman NJ. Blood pressure threshold for abnormal ocular fundus findings is lower than expected. Hypertension 2012;59:e8-e9.

27. Hagen K, Stovner LJ, Vatten L, Holmen J, Zwart JA, Bovim G. Blood pressure and risk of headache: a prospective study of 22685 adults in Norway. J Neurol Neurosurg Psychiatry 2002;72:463-466.

28. Tronvik E, Stovner LJ, Hagen K, Holmen J, Zwart JA. High pulse pressure protects against headache: prospective and cross-sectional data (HUNT study). Neurology 2008;70: 1329-1336.

29. Papadopoulos DP, Mourouzis I, Thomopoulos C, Makris T, Papademetriou V. Hypertension crisis. Blood Press 2010;19: 328-336.

30. Gupta VK. Systemic hypertension, headache, and ocular hemodynamics: a new hypothesis. MedGenMed 2006;8:63.

31. Henderson AD, Bruce BB, Newman NJ, Biousse V. Hypertension-related eye abnormalities and the risk of stroke. Rev Neurol Dis 2011;8:1-9. 